\title{
Die französische EU-Ratspräsidentschaft 2008 - zwischen aktuellem Krisenmanagement und strategischer Weichenstellung
}

\author{
Hans Stark*
}

\begin{abstract}
Wären die von der Gemeinschaft über Jahrzehnte massiv aufgepäppelten Iren nicht erneut der Versuchung erlegen, einen EU-Vertrag per Referendum abzulehnen, hätte am 1. Juli 2008 die letzte ,klassische“ EU-Ratspräsidentschaft begonnen. Die Ironie der Geschichte wollte es, dass die Präsidentschaft von dem Land übernommen wird, dem in erster Linie die Einführung des Europäischen Rates 1974 zu verdanken ist. Nicht zuletzt aus diesem Grund wird in Frankreich der Europapolitik in diesem Jahr eine große Aufmerksamkeit zuteil. Aber das immense Medienecho, das die französische Ratspräsidentschaft an der Seine bereits ausgelöst hat, erklärt sich auch durch andere Gründe. Dem Kreis sowohl der ,großen ' EU-Mitgliedstaaten als auch dem der Gründungsmitglieder zugehörig, geht Frankreich von der Grundannahme aus, dass es über eine im Vergleich zu anderen EU-Mitgliedstaaten historisch und politisch höhere Legitimität verfügt, eine Legitimität, die es ihm zur Aufgabe macht, in Teilbereichen wichtige Akzente zu setzen und der Europäischen Union neuen Schwung zu verleihen.

Frankreich ist sich aber auch der Tatsache bewusst, dass der Erfolg seiner ,Mission“ inzwischen mehr als ungewiss ist. Die ,historische' Legitimität, auf die sich Paris stützen zu können glaubt, hat zum einen durch den negativen Ausgang des EU-Verfassungsreferendums im Mai 2005 in vielen EU-Mitgliedstaaten erheblich an Glaubwürdigkeit eingebüßt. Daran hat auch der Präsidentenwechsel im Élysée Palast im Jahr 2007 sowie der Einsatz Sarkozys für die Unterzeichnung und die reibungslose Ratifizierung des Vertrages von Lissabon in Frankreich wenig geändert. Hinzu kommt die Haltung der französischen Bevölkerung zur Europäischen Union, die, was die Kernfragen zukünftiger Erweiterungen und zunehmender Integration anbelangt, überwiegend skeptisch eingestellt ist. Ein Referendum über den Vertrag von Lissabon hätte mit Sicherheit das gleiche Ergebnis zur Folge gehabt wie das Verfassungsreferendum 2005. ${ }^{1}$ Somit sollte die französische Ratspräsidentschaft auch dazu dienen, die Franzosen mit der Europäischen Union, zu versöhnen“ und an den positiven Grundkonsens wieder anzuknüpfen, der bis in die späten 1980er Jahre vorherrschte.

Dieser pädagogische Aspekt lässt sich aber nur vor dem Hintergrund einer erfolgreichen Ratspräsidentschaft realisieren, die ihrerseits in großem Maße von der Außendarstellung der französischen Europapolitik abhängt. Hier besteht besonderer Handlungsbedarf. Paris wird stets mit dem Vorwurf der ,Arroganz، konfrontiert, was in Frankreich zu einer Fülle von Diskussionen und Stellungnahmen geführt hat. Dass sich die slowenische Regierung attestierte, dass ihre Ratspräsidentschaft zwar nicht so grandios (wie die französische), dafür aber effizient(er) ausfallen wird, passt in dieses Bild von einem Frankreich, das noch immer von vergangener Größe und Führungsmacht träumt, viel verspricht, wenig hält und im Zweifel unilateral agiert.
\end{abstract}

* Dr. Hans Stark, Leiter des Comité d'études des relations franco-allemandes (Cerfa), Institut français des relations internationales (Ifri); Dozent für europäische Geschichte an der Universität Paris 3, Sorbonne Nouvelle.

1 Michel Brulé: Les Français et l'Europe: une question de leadership, in: Sociétal 2/2008, S. 61-65. 


\section{Die Vorbereitungsphase der französischen EU-Ratspräsidentschaft}

Um diese Perzeptionen zu entkräften, ${ }^{2}$ bemüht sich die neue Führung in Paris darum, sowohl in der Form als auch im Inhalt Akzente zu setzen, die sich bewusst von der Europapolitik ihrer Vorgänger abgrenzen. Scheute Frankreich unter Jacques Chirac keine Auseinandersetzung mit den ,kleinen' EU-Mitgliedstaaten, so geht Frankreich heute ganz bewusst auf alle EU-Mitgliedstaaten zu. Präsident Sarkozy und sein Regierungschef François Fillon haben in den letzten Wochen 22 der 26 EU-Partnerländer besucht. ${ }^{3}$ Eine symbolisch herausragende Wirkung hatten die Gipfeltreffen in London und Warschau, die auch dazu dienten, die 2003 bei der Auseinandersetzung um den Irak-Krieg entstandenen Gräben zu überwinden sowie Frankreichs Präsenz in Osteuropa ${ }^{4}$ und die französisch-britische entente formidable, die Sarkozy während seines Besuches in London Ende März 2008 angekündigt hat, ${ }^{5}$ zu unterstreichen. Nicht zuletzt zeugt diese Kontaktaufnahme aber auch von dem Entschluss, nicht mehr zwischen alten und neuen beziehungsweise ,großen' und ,kleinen " EU-Mitgliedstaaten zu unterscheiden und von dem Bemühen um neue bilaterale Allianzen. Diese sollen den von Frankreich inzwischen akzeptierten Bedeutungsverlust des ,deutsch-französischen Motors' in der EU der 27 kompensieren. ${ }^{6}$ Das bedeutet nicht, dass Frankreich etwa mit Polen oder Großbritannien eine Gegenmacht zu Deutschland in der Europäischen Union aufzubauen gedenkt. Aber die Strategie, sich aus der als zu einseitig empfundenen Abhängigkeit von der als zu dominierend wahrgenommenen ,Zentralmacht Deutschland` zu befreien, ist unverkennbar.

Ob das sichtliche Bemühen um neue Partnerschaften ausreichen wird, der französischen Ratspräsidentschaft zum Erfolg zu verhelfen, muss einstweilen dahingestellt bleiben. Die Kampagne der Sympathiewerbung konnte zudem eine Reihe von Unstimmigkeiten zwischen Paris und Brüssel sowie den übrigen EU-Partnern nicht verdecken. Offenen Dissens gibt es erneut in der Wirtschafts- und Währungspolitik, wovon die harsche Kritik Sarkozys an Jean-Claude Trichet und an der Währungspolitik der Europäischen Zentralbank ebenso zeugte, wie die Weigerung des Präsidenten, die Auflagen des Stabilitätspaktes einzuhalten. Die Tatsache, dass die Europäische Kommission Frankreich nur einen Monat vor Beginn seiner Ratspräsidentschaft ,empfohlen ' hat, sein Haushaltsdefizit abzubauen und Strukturreformen in Angriff zu nehmen, ist ein weiterer Hinweis auf die nach wie vor bestehenden Spannungen zwischen Brüssel und Paris. ${ }^{7}$ Auch die Affäre um die bulgarischen Krankenschwestern, die darauf folgende Hofierung Libyens sowie die Parteinahme Frankreichs für den tschadischen Staatschef Idriss Déby während des Putschversuchs in N'Djamena Anfang Februar 2008 haben bei den EU-Partnern Irritationen ausgelöst. ${ }^{8}$

Vor allem aber haben das Ausbrechen Frankreichs aus den Strukturen des Barcelona-Prozesses, die einseitige Ankündigung einer auf die Anrainerstaaten beschränkten aber mit EUMitteln finanzierten Mittelmeerunion und das anschließende, sich über mehrere Monate hin-

2 Agence Reuter: UE - Kouchner promet une présidence française sans arrogance, 13.05.2008.

3 Von diesen Besuchen waren nur die Slowakei, Litauen, Irland (wegen des Referendums) und Slowenien (zu Lubljana gab es ohnehin ständige Kontakte) ausgeschlossen. Vgl. Christophe Schmidt: Présidence française de l'UE: un marathon de visites pour Sarkozy et Fillon, in: Agence France Press, 25.05.2008.

4 Bei seinem Besuch in Warschau hat Sarkozy angekündigt, Frankreichs Grenzen für osteuropäische Arbeitnehmer zu öffnen. Vgl. International Herald Tribune: France to welcome east EU labor, 29.05.2008.

5 Vgl. Andrea Morawski: Sarkozy et Brown lancent "l'Entente formidable“, in: La Tribune, 27.03.2008.

6 Interview mit Jean-Pierre Jouyet: Si la relation franco-allemande est nécessaire, elle n'est pas suffisante, in: Sociétal 2/2008, S. 26-30.

7 Vgl. La Tribune: La Commission européenne tance la France sur son déficit budgétaire, 28.05.2008.

8 Vgl. Frédéric Charillon: Les enjeux de la présidence française de l'Union européenne: opportunités et limites, in: La revue internationale et stratégique 69/2008, S. 161-167. 
ziehende diplomatische Hickhack um dieses Pariser Prestigeobjekt für erhebliche Spannungen sowohl in der Europäischen Union als auch und vor allem zwischen Deutschland und Frankreich gesorgt. Auch wenn auf deutschen Druck hin Mitte März 2008 in dieser Frage ein Kompromiss erzielt werden konnte, ist nicht auszuschließen, dass die ehrgeizige Initiative der Union für das Mittelmeer auch in den kommenden Monaten für Konfliktstoff sorgen wird. Trotz erheblicher Bemühungen um Gespräche und Einflußnahme stand die Vorbereitungsphase der französischen Ratspräsidentschaft somit unter einem ungünstigen Stern. Dass sich Paris dennoch Hoffnungen auf einen positiven Abschluss des zweiten Halbjahrs 2008 macht, hängt jedoch mit der Tatsache zusammen, dass sich Paris nicht nur akribisch auf die Außendarstellung seiner Ratspräsidentschaft vorbereitet hat, sondern vor allem auch auf deren Inhalte.

\section{Vier Hochzeiten (und ein Todesfall?)}

Letztere wurden spätestens Anfang Januar 2008 offiziell bekanntgegeben und auf vier strategisch wichtige Domänen ausgerichtet. So kündigte Präsident Sarkozy in einer Pressekonferenz am 8. Januar 2008 seine Zielsetzung an, dass ,,die EU nach der französischen Ratspräsidentschaft über eine Einwanderungs-, eine Verteidigungs-, eine Energie- und eine Umweltpolitik verfügen werde“. 9 Überraschenderweise hat Sarkozy in diesem Zusammenhang die Tatsache nicht erwähnt, dass die Europäische Union auf diesen vier Feldern bereits über gemeinsame Politiken verfügt, ganz abgesehen davon, dass es sicher vermessen wäre, eine komplette Vergemeinschaftung dieser Bereiche im Laufe einer einzigen Präsidentschaft zu erwarten. Fünfter Schwerpunkt ist die Union für das Mittelmeer, die ursprünglich außerhalb der EU-Strukturen konzipiert war, nun aber durch ihre feierliche Eröffnung am 13. Juli 2008 in Paris unter Mitwirkung aller EU-Mitgliedstaaten fester Bestandteil der französischen Präsidentschaftsagenda geworden ist. Die Wahl der Franzosen ist somit auf fünf hochsensible und für die Zukunft Europas sicher entscheidende Politikfelder gefallen, die sich zwar seit Jahren schon im Fluss befinden, aber zweifellos entwicklungsbedürftig sind beziehungsweise klare Defizite aufweisen.

Die Notwendigkeit einer europäischen Einwanderungspolitik stellt sich spätestens seit der Einführung des Schengener Abkommens 1985 und dessen Ausdehnung auf neun weitere Staaten zu Beginn diesen Jahres. Da die Bekämpfung der illegalen Einwanderung innenpolitisch einen hohen Stellenwert in Frankreich hat, verwundert es nicht, dass sich Paris darum bemüht, die Einwanderungspolitik seiner EU-Partner mit der eigenen in Einklang zu bringen - insbesondere vor dem Hintergrund einer massenhaften Legalisierung illegaler Einwanderer, wie dies etwa in Spanien vorgenommen wurde. Um illegalen Einwanderern die Aufnahme in EU-Europa zu erschweren, plant Frankreich während seiner Ratspräsidentschaft die Verabschiedung eines ,europäischen Einwanderungspaktes' durch die Staats- und Regierungschefs. ${ }^{10}$ Paris plant weiterhin eine Verschärfung der Kontrolle der Außengrenzen, eine Aufwertung der europäischen Grenzschutzagentur Frontex, eine verbesserte Abschiebepraxis illegaler Einwanderer durch effektivere Rückführungsabkommen mit Drittstaaten sowie härtere Bedingungen für legale Einwanderer beziehungsweise deren stärkere Kontrolle (Visa sollen ab 2011 nur noch mit biometrischen Daten gewährt werden), und schließlich plädiert Frankreich auch für eine gemeinsame Asylpolitik. ${ }^{11} \mathrm{Ob}$ dieser Vorstoß den ge-

9 Vgl. Agence Europe: Nicolas Sarkozy se fait l'avocat d'une Europe protectrice, 09.01.2008, S. 6.

10 Vgl. Philippe Setton: Les enjeux de la présidence française du Conseil de l'Union européenne en matière migratoire, in: Regards sur l'actualité 340/2008, S. 29-39.

11 Fidelius Schmid: Sarkozy will Europa abschotten, in: Financial Times Deutschland, 29.05.2008. 
wünschten Erfolg haben wird, ist noch ungewiss. Einwanderungspolitik ist nicht nur ein politisch sehr sensibler Bereich, sondern auch fester Bestandteil der nationalen Zuständigkeit der EU-Mitgliedstaaten, die zudem nicht alle über das gleiche demographische Wachstum wie Frankreich verfügen und zum Teil auch eine grundsätzlich andere Haltung als Frankreich gegenüber der Problematik legaler und illegaler Einwanderung einnehmen.

Ähnlich ehrgeizige Ziele verfolgt Sarkozy in der Sicherheits- und Verteidigungspolitik, die zu den traditionell von Frankreich bevorzugten EU-Politikfeldern gehört. Paris plädiert für konkrete Zielvorgaben, die für alle Mitgliedstaaten verpflichtend sein sollen, um die zivilen und militärischen Kapazitäten des europäischen Krisenmanagements zu erhöhen. Konkret schwebt Frankreich die Schaffung einer europäischen Marine-Luftfahrt-Gruppe oder eine gemeinsame Flotte aus Militärflugzeugen des Typs A400M für den strategischen Truppen- und Materialtransport vor. Unter anderem wünscht sich Frankreich auch einen gemeinsamen Markt für die Rüstungs- und Verteidigungsindustrie und eine Aufwertung der Europäischen Verteidigungsagentur. Paris spricht sich für ein koordiniertes Vorgehen bei der Evakuierung von EU-Bürgern aus Drittstaaten aus, für eine Zusammenlegung der Verteidigungsausgaben einzelner EU-Mitgliedstaaten und eine Konkretisierung des Konzeptes der permanenten strukturierten Zusammenarbeit. Des Weiteren setzt sich Frankreich, das im Juni 2008 ein neues Weißbuch veröffentlicht hat, für eine Überarbeitung der europäischen Sicherheitsstrategie ein. Aus Pariser Sicht muss sie an die seit 2003 durch die EU-Osterweiterung, die Einführung der Europäischen Nachbarschaftspolitik sowie insbesondere durch die Entwicklungen in Moskau und Washington eingetretenen geopolitischen Veränderungen angepasst werden. ${ }^{12}$

Hintergrund dieser Initiative ist die anhaltende Unzufriedenheit Frankreichs mit der Bilanz der Europäischen Sicherheits- und Verteidigungspolitik (ESVP) zehn Jahre nach dem französisch-britischen Gipfel von Saint-Malo. ${ }^{13}$ Die Kritik Frankreichs an der ESVP steigerte sich noch im Zusammenhang mit der zögernden Bereitstellung von Soldaten für die Operation „EUFOR Tchad/RCA“ unter der Leitung Frankreichs. Paris kritisiert insbesondere die Zurückhaltung Deutschlands und dessen unzureichende Verteidigungsausgaben sowie die Tatsache, dass Großbritannien sich seit dem Beginn des Irakkrieges praktisch an keiner EU-Mission mehr beteiligt und den institutionellen Ausbau der ESVP nach Kräften behindert, insbesondere im Hinblick auf autonome Planungskapazitäten und den Ausbau der Europäischen Verteidigungsagentur. Einerseits ist sich Frankreich natürlich der Tatsache bewusst, dass diese Hindernisse Berlin und London betreffend nicht von heute auf morgen aus der Welt zu schaffen sind. ${ }^{14}$ Andererseits muss es seinen Ambitionen gegenüber den beiden wichtigsten ESVP-Partnern aber auch keine Zügel mehr anlegen. Der Vorwurf, Paris wolle gegen den Willen der Mehrheit der EU-Mitgliedstaaten die ESVP zum Zwecke der, Einhegung' amerikanischer Macht instrumentalisieren, ist durch die angekündigte Rückkehr Frankreichs in die integrierte militärische Struktur der NATO haltlos geworden und dient Deutschland und Großbritannien somit auch nicht mehr als Alibi, um die eigenen Unzulänglichkeiten im Rahmen der ESVP zu kaschieren.

In der Energiepolitik nimmt Frankreich traditionell eine Sonderstellung ein. Auch wenn es vom erneuten Ölschock natürlich genauso betroffen ist wie seine Partner, verfügt Frankreich als zweitgrößte Atommacht über eine größere Unabhängigkeit in der Energiepolitik

12 Agence Europe: La présidence française va rouvrir le chantier de la défense européenne, 05.06.2008, S. 6.

13 Vgl. Hans Stark: France and ESDP, in: Bertelsmann Stiftung (Hrsg.): The Big 3 and ESDP. France, Germany and the United Kingdom, European Foreign and Security Policy Nr. 5, Gütersloh 2006, S. 11-21.

14 Interview mit Nicole Gnesotto: La défense européenne comme priorité de la présidence française, in: La revue internationale et stratégique 69/2008, S. 153-159. 
(Frankreich verfügt über 58 der 440 weltweit existierenden Atomanlagen) und sieht dementsprechend in der Atomenergie das entscheidende Instrument, um eine Diversifizierung und Sicherheit der Versorgung zu gewährleisten. Erdgas wird hauptsächlich aus Algerien bezogen, was Frankreich, anders als Deutschland, von Russland energiepolitisch unabhängig macht. Dennoch setzt sich Frankreich für ein gemeinsames Vorgehen und verstärkte Transparenz in den Bereichen, Versorgungssicherheit' gegenüber Drittstaaten ein. In diesem Zusammenhang plädiert Frankreich für den Abschluss eines strategischen Partnerschaftsabkommens zwischen der Europäischen Union und Russland, das insbesondere auf die Problematik der Energieversorgung zugeschnitten sein soll. ${ }^{15}$ Eingeschränkt wird die auf Europa ausgerichtete Energiepolitik durch Frankreichs Streit mit Brüssel über das dritte Energiepaket der Kommission. Ebenso wie Deutschland (und andere EU-Mitgliedstaaten) lehnt Frankreich die Pläne der Kommission ab, eine funktionale Trennung der Erzeugungsund Verteilungsaktivitäten von Energiekonzernen vorzunehmen (die sogenannte, eigentumsrechtliche Entflechtung'). Paris befürwortet einen ,flexibleren' Ansatz, der darin besteht, dass Regulierungsbehörden energieproduzierende Unternehmen nur ,bitten ' könnten, ihre Übertragungsleitungen aufzugeben und Netz- und Infrastrukturverbesserungen in Angriff zu nehmen.

Aus französischer Sicht gehört Atomkraft, ebenso wie die Wind- und Sonnenenergie, zu den erneuerbaren Energiequellen und sollte auch einen entsprechenden Platz im Kampf gegen den Klimawandel einnehmen. Der hohe Anteil der Atomkraft am Energieverbrauch in Frankreich erleichtert es dem Land erheblich, die im März 2007 beschlossenen EU-Klimaschutzziele einzuhalten und die $\mathrm{CO}_{2}$-Emissionen landesweit um 20 Prozent zu reduzieren. So verwundert es nicht, dass Frankreich den Umweltschutz zu einer nationalen Priorität erklärt hat (im Rahmen der Vereinbarung „Grenelle de l'environnement“) und auch die Europäische Union zu einem weltweiten Vorbild für Umweltschutz zu machen gedenkt. ${ }^{16}$ In diesem Zusammenhang beabsichtigt Frankreich, seine Ratspräsidentschaft zu nutzen, um Einfluss auf die kommende UN-Klimakonferenz auszuüben, die im Dezember 2008 in Polen stattfinden wird und auf der der Abschluss eines Nachfolgeabkommens für das KyotoProtokoll angestrebt wird. Des Weiteren wird unter der französischen Ratspräsidentschaft eine Einigung über das ,Energie- und Klimapaket“ erwartet, das die Kommission mit dem Ziel einer Reduzierung der Treibhausgasemissionen bis 2020 um 20 Prozent im Januar 2008 vorgelegt hat. Die Grenze des Erträglichen wird allerdings, wie auch in der Energiepolitik, aus französischer Sicht dann überschritten, wenn Klimaschutzmaßnahmen, die auf EUEbene beschlossen wurden, der Wettbewerbsfähigkeit französischer Unternehmen zu schaden drohen. Vor diesem Hintergrund ist auch der Anfang Juni 2008 erfolgte deutsch-französische Kompromiss über die Klimaschutzvorgaben für die Autoindustrie zu sehen, der den Industrieinteressen beider Länder, und insbesondere Deutschlands, erheblich entgegenkommt. ${ }^{17}$

Unvollständig wäre dieses Panorama ohne den Hinweis auf das französische Projekt einer Mittelmeerunion, das ursprünglich nicht zu den angekündigten Schwerpunkten gehörte, letztere aber mittlerweile doch arg an den Rand gedrückt hat. Von dem ursprünglichen Projekt, einer Union der Mittelmeeranrainerstaaten ohne Beteiligung der nördlichen und östlichen EUMitgliedstaaten, ist dank deutschen Drucks nicht viel übrig geblieben. Es handelt sich beinahe

15 Agence Reuter: La France veut faire avancer le partenariat UE-Russie, 29.05.2008.

16 Vgl. Jean Lamy: Les enjeux de la présidence française du Conseil de l'Union Europénne en matière d'énergie et de lutte contre le changement climatique, in: Regards sur l'actualité 340/2008, S. 17-27.

17 Nico Fried/Cerstin Gammelin: Durchbruch im Abgase-Streit, in: Süddeutsche Zeitung, 09.06.2008. 
um einen Todesfall. Denn der Anfang März 2008 erreichte Kompromiss - eine sich auf konkrete Projekte ${ }^{18}$ beschränkende Union für das Mittelmeer im Rahmen des bestehenden und von Frankreich heftig kritisierten Barcelona-Prozesses - bleibt weit hinter den von Sarkozy ursprünglich anvisierten Zielen zurück. ${ }^{19}$ Insbesondere die Kommission hat der von Frankreich im Rahmen der Union für das Mittelmeer gewünschten Führungsrolle der südlichen und nördlichen Mittelmeeranrainerstaaten einen Riegel vorgeschoben. In ihrer Mitteilung vom 20. Mai 2008 regte die Europäische Kommission an, die zukünftige Ko-Präsidentschaft der Union für das Mittelmeer nicht einem EU-Mittelmeerstaat zu übertragen, sondern bei den für die Außenbeziehungen zuständigen Gemeinschaftsstrukturen anzusiedeln. ${ }^{20}$ Auch bei der Frage der Lokalisierung des zukünftigen Sekretariats der Union für das Mittelmeer könnte die Wahl auf Brüssel und nicht, wie von Frankreich schon inoffiziell angekündigt, auf Tunis fallen. Schwer in diesem Zusammenhang wiegt die Tatsache, dass das Kommissionspapier sowohl von der Mehrheit der EU-Mitgliedstaaten als auch vom Europäischen Parlament begrüßt wurde. ${ }^{21}$ Vor allem aber leidet das gesamte Projekt, wie übrigens auch der zurecht von Paris kritisierte Barcelona-Prozess, unter anderem an der ungelösten Palästina-Frage und der damit zusammenhängenden Nichtanerkennung Israels durch seine Nachbarn sowie am anhaltenden Autoritarismus und der wirtschaftlichen Rückständigkeit der südlichen Mittelmeerstaaten. Diese sind vielmehr an gesonderten bilateralen Beziehungen mit der Europäischen Union als an vertieften Handelsbeziehungen untereinander interessiert - ganz zu schweigen von der demographischen Situation dieser Länder und dem daraus folgenden Migrationsdruck, vor dem sich Frankreich ja gerade durch eine zunehmend repressive Einwanderungspolitik zu schützen versucht. So könnte sich die Union für das Mittelmeer als eine Totgeburt entpuppen, die Paris erneut dazu antreiben könnte, wieder auf die unilaterale Karte zu setzen.

\section{Ausblick}

Frankreich tritt seine Ratspräsidentschaft unter höchst schwierigen Umständen an. Zum einen hat sich Paris sehr hohe, vielleicht sogar zu hohe Ziele gesetzt. Zum anderen trägt das Nein der Iren vom 12. Juni 2008 zu einer erheblichen Verwirrung bei, was den weiteren Verlauf des Ratifikationsprozesses und das Inkrafttreten des Lissabonner Vertrages betrifft. Beides sollte ursprünglich ja unter der französischen Ratspräsidentschaft geklärt werden. Frankreich muss daher nun eine EU-Krise meistern, die jener ähnelt, die das französische Nein 2005 provoziert hat. Gleichzeitig müssen aber noch im zweiten Halbjahr 2008 eine Reihe von Weichen für Entscheidungen gestellt werden, die 2009 anstehen (sollten). Dies betrifft in erster Linie die Reform der Gemeinsame Agrarpolitik und die Neuausrichtung des kommenden EU-Budgets, aber auch wichtige Personalentscheidungen in Bezug auf den Vorsitz der Kommission, den Hohen Vertreter für die Gemeinsame Außen- und Sicherheitspolitik aber auch, im Vorgriff auf den Lissabonner Vertrag, die Kandidatenauswahl für das neue Amt des Präsidenten des Europäischen Rates. Frankreich (und die Europäische Union) stehen vor enormen Herausforderungen, aber wie Napoléon schon zu sagen pflegte, ,l'impossible n'est pas français‘.

18 Im Mittelpunkt der Union für das Mittelmeer stehen insbesondere die Sanierung des Mittelmeers, die Festlegung schneller maritimer Handels- und Transportrouten, die Zusammenarbeit im Bereich der Solarenergie sowie eine intensivere Wissenschaftskooperation.

19 Vgl. Daniela Schwarzer/Isabelle Werenfels: Formelkompromiss ums Mittelmeer, in: SWP-Aktuell 24/2008.

20 Agence Europe: La Commission répond au Conseil européen et dilue totalement le Projet français d'Union pour la Méditerrannée, 21.05.2008, S. 4.

21 Vgl. Agence Europe: In hushed voices partner countries support Commission against Paris but regret absence of genuine concertation, 27.05.2008, S. 8; Agence Europe: Draft resolution on Union for Mediterranean, 03.06.2008, S. 4. 